\title{
Spatial regulation of gene expression in nonsyndromic sagittal craniosynostosis
}

\author{
${ }^{*}$ Garrett N. Cyprus, BS, ${ }^{1}$ Jefferson W. Overlin, BS, ${ }^{1}$ Rafael A. Vega, MD, PhD, ${ }^{2}$ Ann M. Ritter, MD, ${ }^{2}$ \\ and René Olivares-Navarrete, DDS, $\mathrm{PhD}^{1}$
}

\begin{abstract}
'Department of Biomedical Engineering, School of Engineering, Virginia Commonwealth University; and 'Department of Neurosurgery, School of Medicine, Virginia Commonwealth University, Richmond, Virginia
\end{abstract}

\begin{abstract}
OBJECTIVE Cranial suture patterning and development are highly regulated processes that are not entirely understood. While studies have investigated the differential gene expression for different sutures, little is known about gene expression changes during suture fusion. The aim of this study was to examine gene expression in patent, fusing, and fused regions along sagittal suture specimens in nonsyndromic craniosynostosis patients.

METHODS Sagittal sutures were collected from 7 patients (average age 4.5 months) who underwent minimally invasive craniotomies at the Children's Hospital of Richmond at VCU under IRB approval. The sutures were analyzed using micro-CT to evaluate patency. The areas were classified as open, fusing, or fused and were harvested, and mRNA was isolated. Gene expression for bone-related proteins, osteogenic and angiogenic factors, transforming growth factor- $\beta$ (TGF- $\beta$ ) superfamily, and Wnt signaling was analyzed using quantitative polymerase chain reaction and compared with normal sutures collected from fetal demise tissue (control).
\end{abstract}

RESULTS Micro-CT demonstrated that there are variable areas of closure along the length of the sagittal suture. When comparing control samples to surgical samples, there was a significant difference in genes for Wnt signaling, TGF- $\beta$, angiogenic and osteogenic factors, bone remodeling, and nuclear rigidity in mRNA isolated from the fusing and fused areas of the sagittal suture compared with patent areas $(p<0.05)$.

CONCLUSIONS In nonsyndromic sagittal craniosynostosis, the affected suture has variable areas of being open, fusing, and fused. These specific areas have different mRNA expression. The results suggest that BMP-2, FGFR3, and several other signaling pathways play a significant role in the regulation of suture fusion as well as in the maintenance of patency in the normal suture.

https://thejns.org/doi/abs/10.3171/2018.6.PEDS18229

KEYWORDS craniosynostosis; micro-CT; Wnt signaling; TGF- $\beta$ superfamily; craniofacial

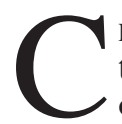

RANIOSYNOSTOSIS, a highly heterogeneous condition characterized by premature ossification of one or more cranial sutures, occurs in approximately one in 2500 live births. ${ }^{12}$ Nonsyndromic craniosynostosis accounts for $75 \%$ of cases of primary craniosynostosis, usually occurring in isolation and typically affecting one suture. ${ }^{3,4}$ In $40 \%-55 \%$ of nonsyndromic cases, the sagittal suture is affected. ${ }^{4}$ It was once thought that primary craniosynostosis was an in utero event, with the entire suture closing at birth. Micro-CT studies have suggested that the patency of the sagittal suture is variable along its course, and gross evaluation during surgical suturectomy would substantiate this finding. A definitive cause of this variable closure is unknown, but spatiotemporal regulation of osteoinductive and osteoinhibitory signaling molecules has been shown to determine the patency or fusion of regions within the cranial sutures and may play a critical role in the development of nonsyndromic craniosynostosis. , $^{4,10,11,17}$

Early craniosynostosis studies demonstrated that the underlying dura played a paracrine signaling role in production and maintenance of sutures at their correct locations over the cranial vault. ${ }^{8}$ Later work found that dif-

ABBREVIATIONS MSC = mesenchymal stem cell; $q \mathrm{PCR}=$ quantitative polymerase chain reaction; TGF $-\beta=$ transforming growth factor- $\beta$.

SUBMITTED April 17, 2018. ACCEPTED June 13, 2018.

INCLUDE WHEN CITING Published online September 14, 2018; DOI: 10.3171/2018.6.PEDS18229.

* G.N.C. and J.W.O. contributed equally to this work. 
ferent isotypes of transforming growth factor- $\beta$ (TGF- $\beta$ ) were present in fused and patent regions of cranial sutures; results suggested that TGF- $\beta 2$ played an osteoinductive role, while TGF- $\beta 3$ played an inhibitory role in cranial sutures. ${ }^{10,11}$ More recently, we showed that osteoinductive BMPs are highly expressed throughout sutural bone, but various inhibitors of BMP signaling (Noggin, Gremlin-1/2, NBL1) were downregulated in prematurely fused regions, suggesting that spatiotemporal disinhibition of osteogenic signaling might contribute to nonsyndromic craniosynostosis. ${ }^{17}$ However, is it not known why or how these changes in BMP inhibition occur.

In addition to dural influence and signaling by TGF$\beta$ superfamily members, Wnt signaling plays a critical role in bone homeostasis and formation. When canonical Wnt ligands bind low-density lipoprotein receptor-related protein (LRP) and Frizzled (Fzd) coreceptors, $\beta$-catenin accumulates intracellularly and translocates inside the nucleus to activate the T-cell factor/lymphoid enhancerbinding factor $(\mathrm{TCF} / \mathrm{LEF})$ transcription factor complex. When Wnt ligands bind to Fzd coreceptors that act independently of $\beta$-catenin, noncanonical signaling occurs. During osteoblastogenesis, both canonical signaling and noncanonical signaling are thought to play synergistic roles in driving mesenchymal stem cell (MSC) differentiation into osteoblasts and preventing differentiation into adipocytes, respectively. ${ }^{9}$ Furthermore, loss-of-function mutations in LRP-5, the coreceptor for canonical Wnt signaling, have been shown to decrease bone mass in both animals and humans.? Conversely, Dickkopf-related proteins (DKKs), sclerostin, and Wnt inhibitory factor (WIF) function as antagonists of Wnt signaling in bone. Studies of Wnt inhibitor expression in closed and patent murine sutures revealed that Wnts and Wnt-associated proteins differentially regulate posterofrontal suture closure and maintenance of the open sagittal suture. ${ }^{2}$

In addition to the aforementioned influences on suture closure, previous studies have implicated mechanical force on sutures in utero as an etiological factor in the development of nonsyndromic craniosynostosis; these mechanical forces are sensed by cells by different "mechanotransducer" proteins affecting gene expression and in some cases resulting in cell differentiation. ${ }^{6,14}$ Unfortunately, little is known about the role or expression of these proteins in normal suture patency and fusion. Among these proteins, integrins, integrin-associated proteins such as Rho small GTPases, linker of nucleoskeleton and cytoskeleton (LINC) complex proteins, intranuclear proteins such as Lamin A/C, and Hedgehog represent potential mechanistic players in the transduction of mechanical force into osteogenic activity at the sutural bone front. Fetal constraintinduced craniosynostosis in rodents has been associated with downregulation of BMP and Indian Hedgehog (IHH) signaling, ${ }^{5}$ but this has not yet been observed in human craniosynostosis.

In this study, we collected bone samples from minimally invasive sagittal suturectomies in pediatric patients with nonsyndromic craniosynostosis and, using micro-CT, identified patent, fusing, and fused sagittal suture regions within the same suture. Using quantitative polymerase chain reaction (qPCR), we measured the expression of genes associated with angiogenesis, TGF- $\beta$ superfamily signaling, Wnt signaling, osteogenesis, and mechanotransduction. Differential expression of genes within each of these classes was found in the patent, fusing, and fused sagittal suture regions, suggesting significant roles in the maintenance of patency or regulation of fusion in the human suture.

\section{Methods \\ Bone Collection}

Bone samples were collected from pediatric donors (5 males and 2 females; average age 4.5 months, range 2-6 months) undergoing minimally invasive corrective surgery for nonsyndromic sagittal craniosynostosis at the Children's Hospital of Richmond at VCU. Institutional review board approval was obtained from Virginia Commonwealth University, with written consent from the donor's parent or guardian. Samples obtained were confirmed nonsyndromic craniosynostosis without any other craniofacial or genetic disease. Sagittal sutures from fetal demise donors ( $\mathrm{n}=4$, mean age 32 weeks) were used as controls. Samples were immediately stabilized in RNAlater (ThermoFisher Scientific) after suturectomy and stored at $-20^{\circ} \mathrm{C}$ until analyzed. Bones were examined by microCT using a voxel size of $11 \mu \mathrm{m}$ (SkyScan 1172, Bruker microCT). Sutures were cleaned from any soft tissue adjacent to the suture. Using micro-CT images, regions of suture samples were identified as patent if the space from the internal face to external face was unoccupied by bone, fusing if the internal edge was fused but the external was not, and fused if the suture space was entirely occupied by bone.

\section{RNA Isolation and qPCR Analysis}

Tissues were minced and placed in TRIzol (ThermoFisher Scientific). RNA was extracted according to the manufacturer's protocol. RNA was reverse transcribed into cDNA with a High Capacity cDNA Kit (Life Technologies). Messenger RNA levels were measured using a custom PCR array (Bio-Rad) designed for osteogenic and bone-related proteins, transforming growth factor superfamily members, and Wnt ligands and associated proteins (Table 1). Three housekeeping genes were measured $(G A P D H, R P S 18$, and TBP) and used to normalize data.

\section{Statistical Analysis}

Experiments were conducted with 4 replicates from each region (patent, fusing, and fused) of each suture and control sutures. RNA expression was analyzed using the $\Delta \Delta \mathrm{Ct}$ method normalized over housekeeping genes and control sutures. Prism GraphPad V7 software was used to analyze the results. Data were found to be normally distributed based on analysis of QQ plots for each group. A one-factor, equal-variance ANOVA was used to test the null hypothesis that the group means were equal, against an alternative hypothesis that at least 2 of the group means were different at a significance level of $\alpha=0.05$. Once the $\mathrm{p}$ value resulting from the initial ANOVA model was determined to be $<0.05$, multiple comparisons were made between group means with Tukey HSD. Plots of the fold 
Cyprus et al.

TABLE 1. Genes included in the custom PCR array

\begin{tabular}{|c|c|c|c|c|c|c|c|c|c|c|c|}
\hline \multirow{2}{*}{$\begin{array}{c}\text { Bone-Related Genes } \\
\text { ALPL }\end{array}$} & \multicolumn{3}{|c|}{ TGF- $\beta$ Superfamily } & \multicolumn{2}{|c|}{ Wnt Ligands } & \multicolumn{3}{|c|}{ Wnt-Associated Proteins } & \multicolumn{2}{|c|}{ Other } & \multirow{2}{*}{$\begin{array}{c}\text { Housekeeping } \\
\text { GAPDH }\end{array}$} \\
\hline & ACVR1 & TGFB2 & SOST & WNT1 & WNT10B & FZD1 & FZD10 & DVL3 & $B C L 11 B$ & LMNA & \\
\hline$B G L A P$ & BMPR1A & TGFB3 & & WNT3A & WNT11 & FZD2 & KREMEN1 & AXIN2 & SPHKAP & RHOA & RPS18 \\
\hline DMP1 & BMPR1B & TGFBR1 & & WNT4 & WNT16 & FZD3 & LRP5 & CTNNB1 & NELL1 & SUN2 & TBP \\
\hline$I B S P$ & BMPR2 & TGFBR2 & & WNT5A & & FZD4 & LRP6 & $D K K 1$ & $R A R A$ & SYNE2 & \\
\hline PTH & BMP2 & CER1 & & WNT5B & & FZD5 & ROR2 & DKK2 & $R A R B$ & TAZ & \\
\hline RUNX2 & BMP4 & $C H R D$ & & WNT7A & & FZD6 & VANGL1 & SFRP1 & $R A R G$ & YAP1 & \\
\hline SP7 & BMP6 & DAND5 & & WNT7B & & FZD7 & VANGL2 & SFRP2 & $\mathrm{IHH}$ & & \\
\hline TNSFS11 & BMP7 & GREM1 & & WNT9B & & FZD8 & DVL1 & WIF1 & SHH & & \\
\hline TNFRSF11B & TGFB1 & NOG & & WNT10A & & FZD9 & DVL2 & & DAAM1 & & \\
\hline
\end{tabular}

change and statistical significance $(-\log 10)$ were used to identify highly regulated genes.

\section{Results}

To identify regions of the patent, fused, and fusing suture, samples taken from infants with nonsyndromic sagittal craniosynostosis (Fig. 1A) or from fetal demise (controls; Fig. 1B) were imaged using micro-CT and reconstructed into 3D models. This analytical method revealed a characteristic pattern of suture closure in synostotic samples where the internal face of the calvaria fused first, suggesting that the fusion front starts at the internal edges of the suture and moves externally.

Next, differences in expression of 90 genes known to play a role in calvarial bone formation and homeostasis were measured in samples of patent, fusing, and fused sutural bone using custom qPCR arrays and normalized to expression in sutures from fetal demise tissue (Fig. 2A). Twelve genes were significantly upregulated in the patent suture, 38 in the fusing suture, and 44 in the fused suture from synostotic samples (Fig. 2B-D). Although least different from control, changes in gene expression in the patent suture from synostotic samples suggest a suturewide change during craniosynostosis - not just at fusing or fused sites within the suture.

Genes associated with osteoblastic differentiation
(ALPL, BGLAP, DMP1, IBSP, RUNX2, and SP7) were evaluated first and were found to be upregulated primarily in the fusing or fused suture (Fig. 3). The gene encoding receptor activator of nuclear factor- $\mathrm{\kappa B}$ ligand (RANKL) (TNFSF11), a promoter of osteoclastogenesis and subsequent bone resorption, was upregulated in patent bone, while the gene encoding RANKL decoy receptor osteoprotegerin $(T N F S F 11 B)$ was upregulated in the fused suture. These results suggest increased MSC-to-osteoblast differentiation in the synostotic suture.

Next, expression of genes in the TGF- $\beta$ superfamily was quantified. Of these, $B M P 2$ was higher in fused and fusing portions of the suture than in the open suture (Fig. 3). Expectedly, mRNA encoding BMP antagonists Noggin (NOG) and Gremlin-1 (GREMI) increased in the patent suture compared with the fusing and fused suture. ${ }^{17}$ $D A N D 5$ and $C H R D$, which encode BMP signaling antagonists, were upregulated in the fused suture compared with both the fusing and patent suture. Of the genes encoding TGF- $\beta$ and its multimeric receptor, mRNA for osteoinductive TGF- $\beta 2$ (TGFB2) was significantly upregulated in fusing bone, while $T G F B R 2$ was significantly upregulated in the patent suture.

Expression of mRNA for Wnt ligands and Wnt signaling-associated proteins was then characterized. Ligands WNTI, WNT5A, and WNT11 were upregulated the most in the fusing and fused suture compared with the patent
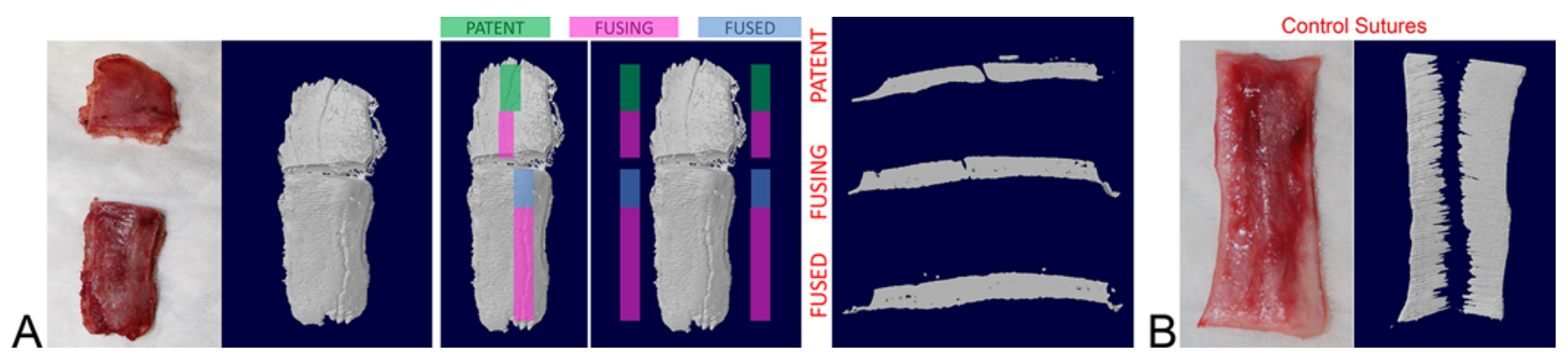

FIG. 1. Reconstruction of sagittal suturectomy tissue samples using micro-CT. Samples were collected by minimally invasive suturectomy. A: Regions of suture from synostotic samples $(n=7)$ were classified as patent (green) if there was a continuous space from the internal to external face of the suture, fusing (magenta) if the internal face was fused but the external was not, or fused (blue) if the region was fully fused from the internal to external face. B: Control samples, collected from deceased fetuses $(n=4)$, were all patent. Figure is available in color online only. 

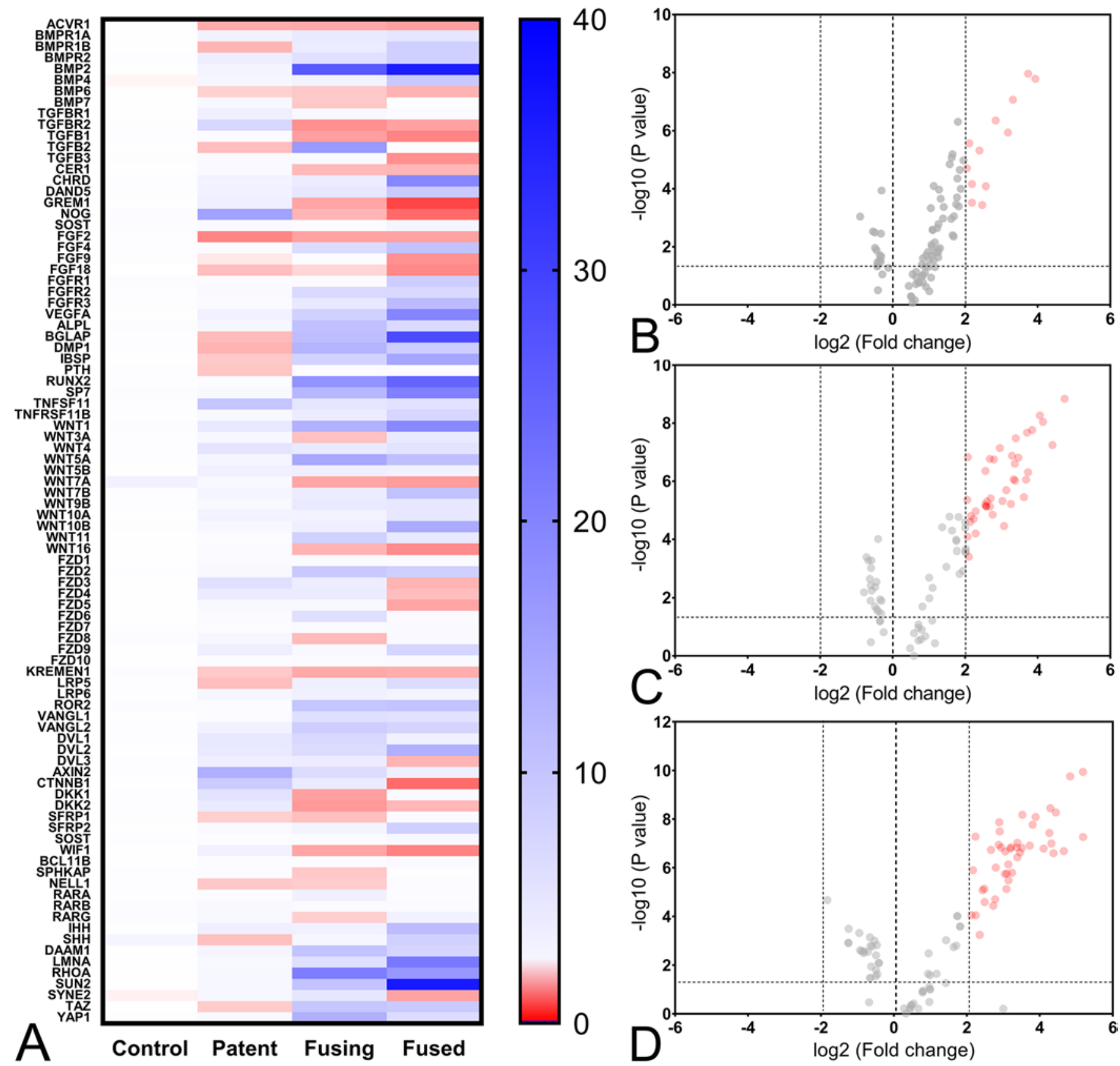

FIG. 2. Differential expression of genes associated with calvarial bone formation and homeostasis. Gene expression was analyzed using PCR array. A: Heat map characterizing the magnitude of upregulation of 90 genes with respect to fetal demise controls. B-D: Volcano plots demonstrating the pattern of upregulation of genes in patent (B), fusing (C), and fused (D) craniosynostosis patient samples compared with control; dotted lines indicate threshold of statistical significance. Data are presented as $\log _{2}($ fold change). Figure is available in color online only.

suture (Fig. 4). WNT7B and WNT10B were upregulated more in the fused compared with the fusing or patent suture. FZD2 was significantly upregulated in the fused and fusing suture, $F Z D 9$ was upregulated the most in the fused suture, and FZD6 was upregulated the most in the fusing suture. FZD 3 and FZD4 were most upregulated in the patent and fusing suture. LRP5 was upregulated primarily in the fused and fusing suture. ROR2, VANGL1, and $V A N G L 2$, genes involved in noncanonical Wnt signaling, were upregulated mainly in the fusing and fused suture. Other Wnt-associated genes upregulated primarily in the fused suture included disheveled gene (DVL2) and secreted frizzled-related protein gene (SFRP2). Genes for Wnt signaling inhibitors (DKK1,DKK2, and WIFl) and genes related to canonical Wnt signaling (CTNNB1 and AXIN2) were primarily higher in patent sutures.

Angiogenic genes were differentially regulated during suture fusion. Significant increases in FGF4, FGFRl, 

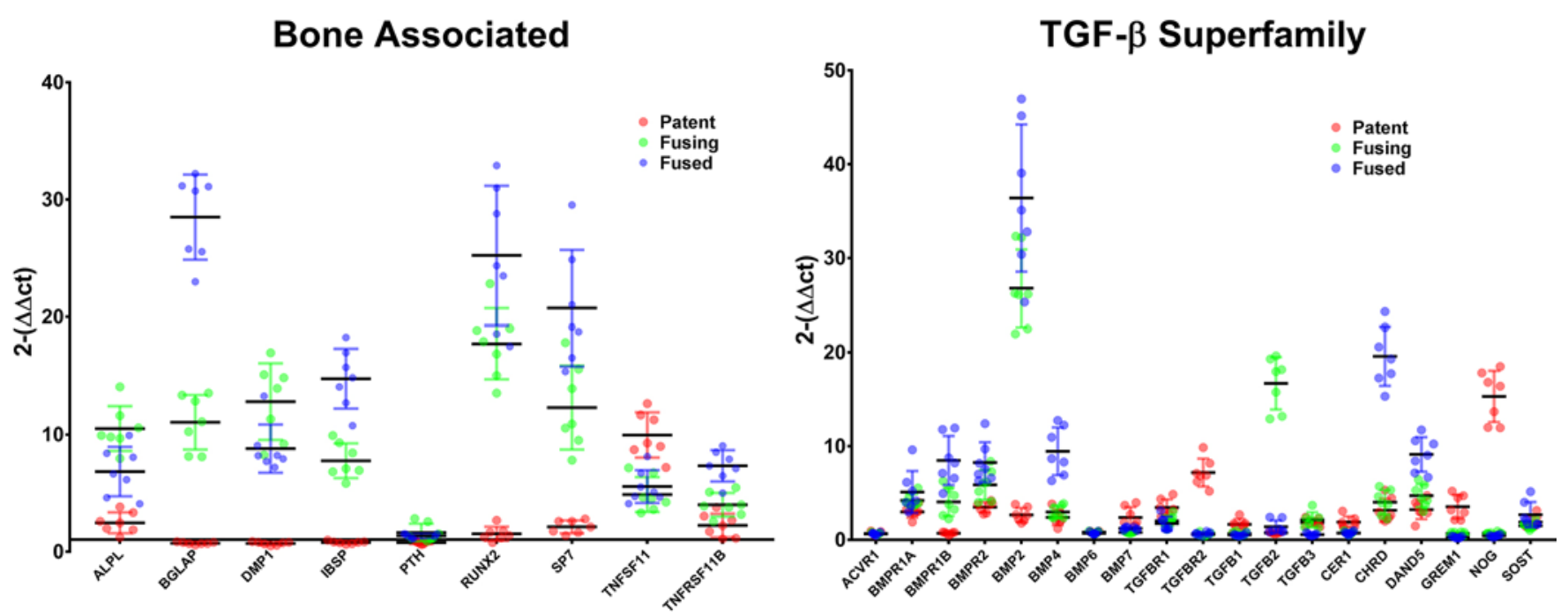

FIG. 3. Upregulation of bone-associated and TGF- $\beta$ superfamily genes in patent, fusing, and fused craniosynostotic samples. Gene expression was evaluated by PCR. Data are presented as fold change $\left(2^{-\Delta \Delta c t}\right)$ with respect to control samples and to respective sample housekeeping gene expression. Figure is available in color online only.

FGFR2, FGFR3, and VEGFA were seen in fusing and fused bone versus patent suture.

Finally, expression of various genes involved in mechanotransduction was measured. Of these, Indian Hedgehog and Sonic Hedgehog genes (IHH and SHH), Lamin $\mathrm{A} / \mathrm{C}$ gene (LMNA), and the LINC complex protein SUN2 (SUN2) were more greatly upregulated in the fused suture than in the fusing or patent suture (Fig. 5). DAAM1, which encodes a scaffolding protein that binds Rho family small GTPases, and RHOA, which encodes Rho GTPase A, were both similarly upregulated in the fusing and fused suture, suggesting a role for integrin signaling in premature suture closure. Genes primarily upregulated in fusing versus patent or fused suture included the gene for retinoic acid receptor- $\alpha(R A R A)$, LINC complex protein SYNE2 (SYNE2), and yes-associated protein 1 (YAPI), the last of which is thought to play a crucial role in cell proliferation in response to cell-cell contact. Together, these findings implicate mechanical stimuli in the process of synostosis.

\section{Discussion}

Proper maintenance of sutural patterning results from a finely tuned balance of promotion and inhibition of bone formation. As we have shown previously, patterns of upregulation of highly osteogenic BMPs and BMP inhibitors

\section{Wnt Ligands}

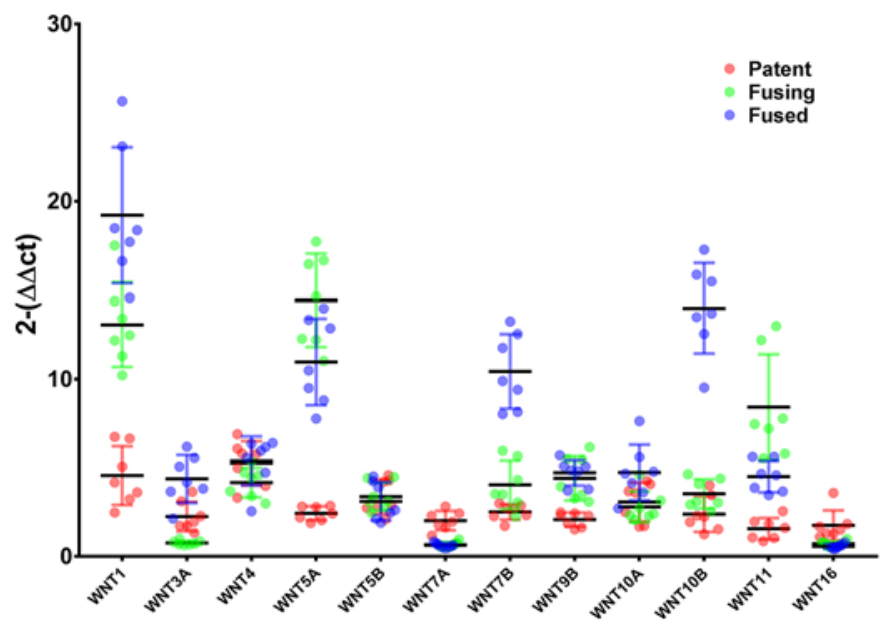

\section{Wnt Associated}

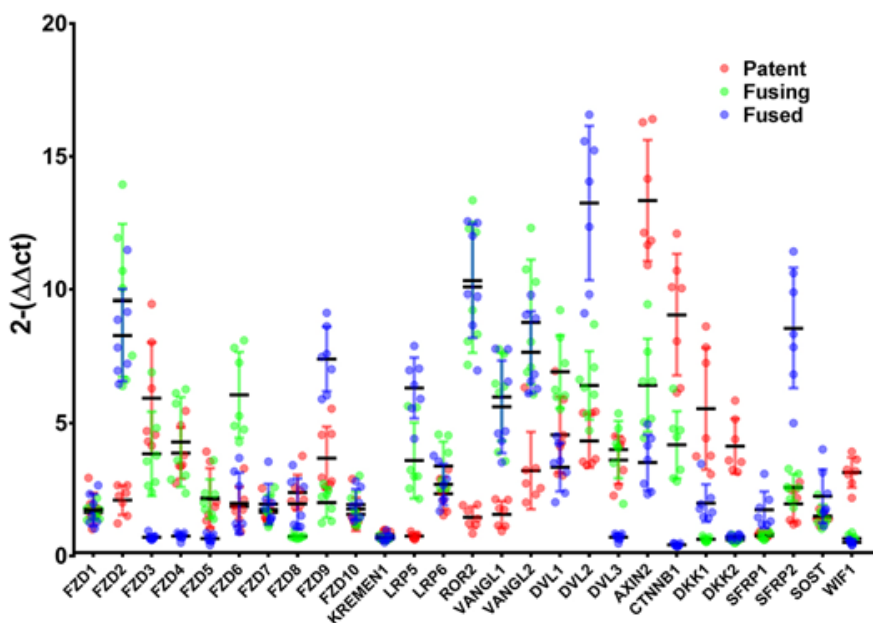

FIG. 4. Differential regulation of Wnt signaling in patent, fusing, and fused craniosynostotic suture. Gene expression was evaluated by PCR. Data are presented as fold change (2- $-\Delta c t)$ with respect to control samples and to respective sample housekeeping gene expression. Figure is available in color online only. 


\section{Blood Vessel Associated}

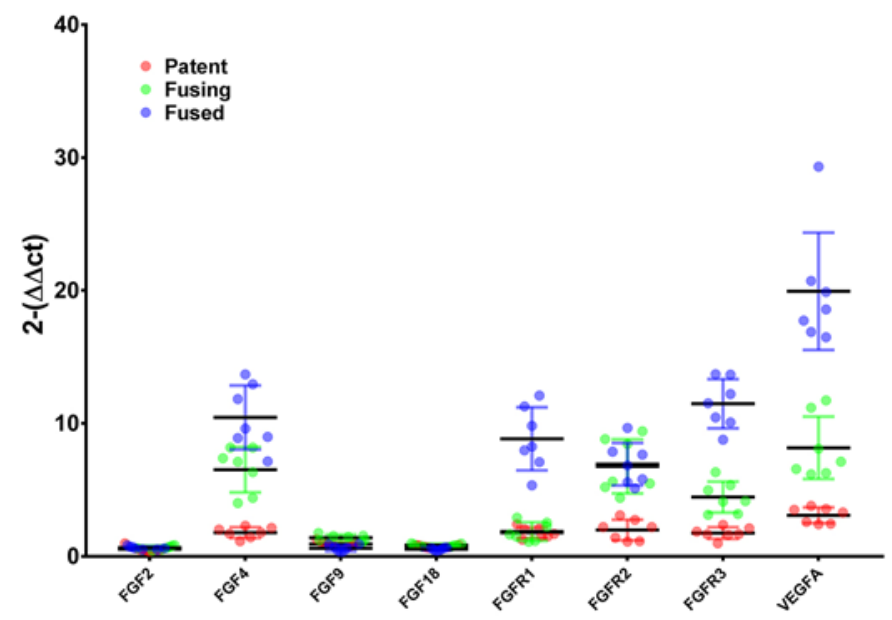

Other

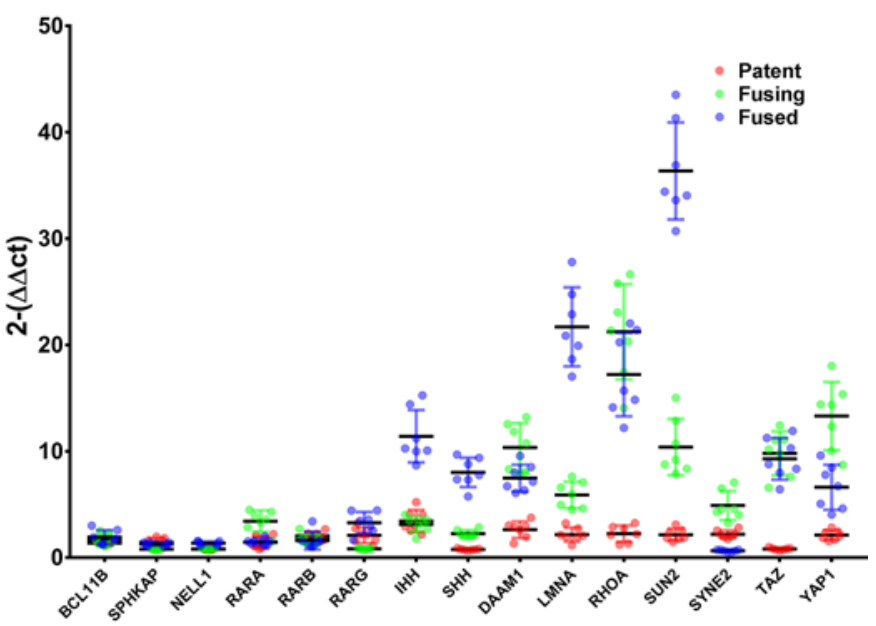

FIG. 5. Upregulation of genes associated with angiogenesis and mechanotransduction in craniosynostotic sagittal suture. Gene expression was evaluated by PCR. Data are presented as fold change $(2-\Delta \Delta c t)$ with respect to control samples and to respective sample housekeeping gene expression. Figure is available in color online only.

reflect patterns of bony and open calvaria, respectively. ${ }^{17}$ In other studies, TGF- $\beta$ isoforms have been shown to exhibit a similar role; in rodent models, TGF- $\beta 3$ promoted sutural patency, while TGF- $\beta 2$ promoted premature closure. ${ }^{10,11}$ We found similar results in this study with respect to BMP signaling, and we did find upregulation of $T G F B 2$ in fusing suture, but TGFB3 was not upregulated in patent suture. However, rodent suture closure differs from human closure in that it occurs by endochondral ossification via differentiation of mesenchymal stem cells into chondrocytes, ${ }^{1}$ since chondrocyte differentiation is known to be highly regulated by TGF- $\beta$ signaling, ${ }^{15,16}$ this difference in human craniosynostosis might support a lesser role of TGF- $\beta$ signaling in human suture closure.

While previous studies of suture formation have identified the importance of the spatiotemporal distribution of BMP and TGF- $\beta$ signaling, little is known about the role of crosstalk between these pathways and others in the context of premature sutural ossification. ${ }^{13}$ Furthermore, Wnt signaling has not been thoroughly explored in human craniosynostosis as a mediator of premature suture closure. In this study, we identified Wnt7B and Wnt10B as exclusively upregulated in the fused suture, and Wnt1 and Wnt5A in both fusing and fused suture. Interestingly, no Wnt ligand was upregulated in the patent suture compared with the fusing or fused suture. Instead, Wnt signaling inhibitors like DKK1/2, WIF1, and Axin2 were upregulated in the patent suture, further supporting that active Wnt signaling is involved in suture closure by promoting osteogenic differentiation of MSCs at the bone front. This interaction is also supported by our findings that gene markers of MSC osteogenic differentiation were upregulated in fusing or fused bone.

While differential TGF- $\beta$ and Wnt gene expression are evident in fused, fusing, and patent bone, these differences do not directly suggest a cause for nonsyndromic craniosynostosis. To explore a mechanical force-based etiology, we measured the gene expression of proteins involved in mechanotransduction. Of these, genes for proteins involved in integrin signaling (DAAM1 and $R H O A)$ were upregulated in fusing and fused bone, suggesting a role for cell-ECM interaction in premature suture fusion. Upregulation of $L M N A$ and $S U N 2$, components of the nuclear side of the LINC complex, occurred primarily in fused bone, and to a lesser extent, in fusing bone; these results underscore an essential role for mechanical stimuli in suture maintenance, as the LINC complex carries mechanical information from the cytoskeleton to the nucleus, which can then induce changes in gene expression.

While craniosynostosis has been explored in animal models, little has been published about the regulation of cranial suture closure in humans because of the circumstances of the disease and affected population. It is uncommon to collect sutural tissue except in cases of craniosynostosis, so there are no readily available age-matched controls. Instead, we used late-gestational fetal demise tissue, which is a limitation in our study. While there is no precedent for its use as a control, we elected to use fetal tissue because age-matched tissue from the sagittal suture is not readily available, and, due to the slow rate of obliteration of the sagittal suture (as late as the 3rd decade of life), we reasoned that there would be little difference between infant and late fetal sutures. Interestingly, in our study, we found the fewest number of dysregulated genes between fetal demise control and synostotic patent samples; these results suggest either a difference due to the condition affecting the entire suture or differences due to age. Nevertheless, the spatial differences in gene expression between open, closing, and closed synostotic suture are apparent in our data.

Craniosynostosis is a relatively common craniofacial anomaly associated with a risk of significant morbidity without treatment. Beyond physical health, correction is often necessary due to the psychosocial consequences of craniofacial malformation during childhood. Due to the risks associated with surgical correction in the pediatric 
population, a deeper understanding of the cellular processes regulating craniosynostosis should be sought in the hope of finding less invasive, preventative treatment earlier in fetal development. Furthermore, exploration of the mechanism of cranial suture closure may contribute to the understanding of bone healing and pathologic bone formation.

\section{Conclusions}

In this study, we sought to characterize the morphometric pattern of pathologic premature suture closure and identify differentially expressed genes in patent, fusing, and fused bone in patients with nonsyndromic sagittal craniosynostosis. First, using micro-CT and 3D reconstruction, we identified an internal-to-external fusing pattern of suture closure. Then, using qPCR, we characterized the gene expression profiles of these 3 classifications of sutural bone. In patent bone, BMP inhibitors, TGF- $\beta$ receptor 2 , Wnt signaling inhibitors, proteins involved in canonical Wnt signaling, and osteoclastogenic RANKL (TNFSF11) were upregulated. In fusing bone, TGFB2, WNT5A, $W N T 11, F Z D 6$, and $Y A P 1$ were upregulated to the greatest extent. Finally, FGFR1, FGFR3, VEGFA, BMP4, CHRD, WNT7B, WNT1OB, DVL2, SFRP2, BGLAP, TNFRSF11B, $I H H, S H H, L M N A$, and SUN2 were upregulated in fused bone. These findings suggest a role for TGF- $\beta$, Wnt, and mechanotransductive signaling pathways in the pathologic closure of the sagittal suture in nonsyndromic craniosynostosis.

\section{Acknowledgments}

This study was supported by the AANS/CNS Pediatric Section Research award and Children's Hospital of Richmond Research Fund.

\section{References}

1. Behr B, Longaker MT, Quarto N: Absence of endochondral ossification and craniosynostosis in posterior frontal cranial sutures of Axin2-/- mice. PLoS One 8:e70240, 2013

2. Behr B, Longaker MT, Quarto N: Differential activation of canonical Wnt signaling determines cranial sutures fate: a novel mechanism for sagittal suture craniosynostosis. Dev Biol 344:922-940, 2010

3. Bennis Y, Wolber A, Vinchon M, Belkhou A, DuquennoyMartinot V, Guerreschi P: [Non syndromic craniosynostosis.] Ann Chir Plast Esthet 61:389-407, 2016 (Fr)

4. Hermann CD, Hyzy SL, Olivares-Navarrete R, Walker M, Williams JK, Boyan BD, et al: Craniosynostosis and Resynostosis. J Dent Res 95:846-852, 2016

5. Jacob S, Wu C, Freeman TA, Koyama E, Kirschner RE: Expression of Indian Hedgehog, BMP-4 and Noggin in craniosynostosis induced by fetal constraint. Ann Plast Surg 58:215-221, 2007

6. Kirschner RE, Gannon FH, Xu J, Wang J, Karmacharya J, Bartlett SP, et al: Craniosynostosis and altered patterns of fetal TGF-beta expression induced by intrauterine constraint. Plast Reconstr Surg 109:2338-2354, 2002

7. Krishnan V, Bryant HU, Macdougald OA: Regulation of bone mass by Wnt signaling. J Clin Invest 116:1202-1209, 2006
8. Levine JP, Bradley JP, Roth DA, McCarthy JG, Longaker MT: Studies in cranial suture biology: regional dura mater determines overlying suture biology. Plast Reconstr Surg 101:1441-1447, 1998

9. Okamoto M, Udagawa N, Uehara S, Maeda K, Yamashita T, Nakamichi Y, et al: Noncanonical Wnt5a enhances Wnt/ $\beta$ catenin signaling during osteoblastogenesis. Sci Rep 4:4493, 2014

10. Opperman LA, Adab K, Gakunga PT: Transforming growth factor- $\beta 2$ and TGF- $\beta 3$ regulate fetal rat cranial suture morphogenesis by regulating rates of cell proliferation and apoptosis. Dev Dyn 219:237-247, 2000

11. Opperman LA, Chhabra A, Cho RW, Ogle RC: Cranial suture obliteration is induced by removal of transforming growth factor (TGF)-beta 3 activity and prevented by removal of TGF-beta 2 activity from fetal rat calvaria in vitro. J Craniofac Genet Dev Biol 19:164-173, 1999

12. Panigrahi I: Craniosynostosis genetics: the mystery unfolds. Indian J Hum Genet 17:48-53, 2011

13. Potter AB, Rhodes JL, Vega RA, Ridder T, Shiang R: Gene expression changes between patent and fused cranial sutures in a nonsyndromic craniosynostosis population. Eplasty 15:e12, 2015

14. Sanchez-Lara PA, Carmichael SL, Graham JM Jr, Lammer EJ, Shaw GM, Ma C, et al: Fetal constraint as a potential risk factor for craniosynostosis. Am J Med Genet A 152A:394400,2010

15. Sueyoshi T, Yamamoto K, Akiyama H: Conditional deletion of Tgfbr 2 in hypertrophic chondrocytes delays terminal chondrocyte differentiation. Matrix Biol 31:352-359, 2012

16. van der Kraan PM, Blaney Davidson EN, Blom A, van den Berg WB: TGF-beta signaling in chondrocyte terminal differentiation and osteoarthritis: modulation and integration of signaling pathways through receptor-Smads. Osteoarthritis Cartilage 17:1539-1545, 2009

17. Whitton A, Hyzy SL, Britt C, Williams JK, Boyan BD, Olivares-Navarrete R: Differential spatial regulation of BMP molecules is associated with single-suture craniosynostosis. J Neurosurg Pediatr 18:83-91, 2016

\section{Disclosures}

The authors report no conflict of interest concerning the materials or methods used in this study or the findings specified in this paper.

\section{Author Contributions}

Conception and design: Olivares-Navarrete, Cyprus, Overlin. Acquisition of data: Cyprus, Overlin, Vega, Ritter. Analysis and interpretation of data: Olivares-Navarrete, Cyprus, Overlin, Ritter. Drafting the article: all authors. Critically revising the article: Olivares-Navarrete, Overlin, Vega. Reviewed submitted version of manuscript: Olivares-Navarrete. Approved the final version of the manuscript on behalf of all authors: Olivares-Navarrete. Statistical analysis: Olivares-Navarrete. Administrative/technical/material support: Overlin, Ritter. Study supervision: Olivares-Navarrete.

\section{Correspondence}

René Olivares-Navarrete: VCU School of Engineering, Virginia Commonwealth University, Richmond, VA. ronavarrete@vcu.edu. 